\title{
Darfour : triste anniversaire
}

\section{Alice Franck et Ivan Deret}

\section{(2) OpenEdition}

Journals

Édition électronique

URL : http://journals.openedition.org/echogeo/3543

DOI : 10.4000/echogeo.3543

ISSN : 1963-1197

\section{Éditeur}

Pôle de recherche pour l'organisation et la diffusion de l'information géographique (CNRS UMR 8586)

\section{Référence électronique}

Alice Franck et Ivan Deret, «Darfour : triste anniversaire », EchoGéo [En ligne], Sur le Vif, mis en ligne le 10 avril 2008, consulté le 05 mai 2019. URL : http://journals.openedition.org/echogeo/3543; DOI :

10.4000/echogeo.3543

Ce document a été généré automatiquement le 5 mai 2019.

\section{(c) (i) (9)}

EchoGéo est mis à disposition selon les termes de la licence Creative Commons Attribution - Pas d'Utilisation Commerciale - Pas de Modification 4.0 International 


\title{
Darfour : triste anniversaire
}

\author{
Alice Franck et Ivan Deret
}

1 En février 2003 un groupe armé s'emparait de la petite ville de Gulu (Nord Darfour), marquant le début d'un cycle de combats et d'une répression sanglante. Cinq années plus tard, la crise du Darfour se poursuit sans qu'aucune issue au conflit ne se dessine.

2 La crise du Darfour surgit dans l'actualité à un moment où l'attention internationale est ailleurs : au Soudan notamment, où elle se concentre sur les avancées des pourparlers de paix entre le gouvernement central et la rébellion du Sud Soudan, qui visent à mettre un terme à l'un des plus longs conflits civils africains ${ }^{1}$. Il faudra attendre la fin de l'année 2003 pour voir s'amorcer une mobilisation de la communauté internationale pour le Darfour.

3 Cette indifférence qui a prévalu durant les premiers mois de la crise est aujourd'hui - fort heureusement révolue: le Darfour, aujourd'hui largement évoqué, est devenu une question prioritaire tant pour les politiques que pour les opinions, unanimement mobilisés. La question du Darfour fait même souvent l'objet de vives polémiques, qui révèlent la multiplicité des approches, et participent à troubler la compréhension d'une crise qui ne cesse par ailleurs de se complexifier.

4 Sans guère d'importance s'ils se limitaient à n'être que déclarations indignées, dénonciations révoltées ou rappels de spécialistes, ces «débats » prennent une toute autre dimension lorsqu'ils influencent l'intervention des différents acteurs humanitaires, diplomates, politiques, voire parties au conflit.

5 Parmi les données ne faisant pas débat, il y a celles-ci : le Darfour est un territoire grand comme l'Espagne, occupant un large quart nord-ouest de l'actuel Soudan et peuplé de plus de 7 millions d'habitants - sur les quelques 33 millions que compte le pays ${ }^{2}$. C'est une région de longue date marginalisée, en proie à des conflits locaux et régionaux, euxmêmes exacerbés par un déséquilibre croissant entre démographie et ressources naturelles, et par diverses instrumentalisations liées aux jeux politiques national et régional. 
Si s'exprime au Darfour l'imbrication d'une "pluralité de causes, d'intérêts et d'échelles » (Denis, 2005), les racines du conflit sont connues. Elles sont à chercher du côté du contexte de sous-développement chronique dont souffre la région occidentale soudanaise depuis son intégration au pays en 1916, sans qu'aucun des gouvernements soudanais successifs n'ait jamais tenté d'y impulser un quelconque développement économique (Lavergne, 2005 ; Prunier, 2005).

7 Replacer le conflit du Darfour dans le contexte soudanais d'injustice régionale et ethnique ${ }^{3}$, et rappeler que la question du partage du pouvoir et des richesses au niveau national est au cœur de la revendication armée des mouvements rebelles du Darfour, n'est pas inutile, cet élément du conflit ayant connu un succès médiatique moins marqué que les lectures plus « simplificatrices» et « sensibles » de la crise.

8 On en oublierait presque aujourd'hui que la répression du gouvernement central, caractérisée par l'emploi de milices « arabes » (janjawid) et une violence inouïe, répondait aussi à des attaques rebelles (prise de Gulu, occupation de Kutum, attaque de l'aéroport d'El Fasher en avril 2003).

9 Le potentiel de déstabilisation du conflit du Darfour sur le gouvernement central est d'autant plus important qu'il concerne une région fortement peuplée, très largement représentée dans l'armée, et qu'il oppose des gens du Nord Soudan, tous musulmans et donc alliés dans l'optique politique courante (Denis, 2005, p. 100).

Dans le cas du Darfour, l'opposition manichéenne entre musulmans et chrétiens, affirmée sans nuance au sujet de la guerre entre le nord et le sud, n'est donc pas valide; s'y substitue néanmoins, avec la force qu'on connait aux visions simplificatrices, le clivage largement repris par la presse, entre « agriculteurs africains » et " pasteurs arabes ». Or, si les dimensions ethnique et foncière sont des composantes essentielles du conflit, la complexité de ces questions ne saurait trouver de traduction dans une telle opposition. Elle ne permet en effet ni de rendre compte du fait qu'il existe des pasteurs non arabes (comme les Zaghawa, fortement représentés dans la rébellion) ou encore des agriculteurs arabes, ni de ce que tous les arabes n'ont pas pris part au conflit quand certaines tribus «Africaines» ont combattu auprès des milices progouvernementales (Tubiana, 2006, p. 112).

11 Plus récente, l'interprétation d'un conflit opposant «musulmans laïcs et/ou modérés » refusant l'ordre incarné par les «musulmans intégristes » de Khartoum, fait peu de cas de la présence active au Darfour d'un groupe rebelle majeur notoirement proche de la mouvance islamiste, le Mouvement pour la Justice et l'Egalité (JEM), mais surtout s'éloigne des réalités du Darfour et du Soudan.

12 A ces lectures schématiques des dynamiques locales vient s'ajouter une approche tout aussi réductrice de la dimension internationale de la crise du Darfour. Ainsi, la question du pétrole - et derrière elle, celle de la responsabilité chinoise - est de plus en plus souvent associée à la crise du Darfour, comme si elle en était, finalement, le véritable enjeu (Vernochet, 2007). Les appels au boycott des jeux de Pékin se multiplient. Or, de quel pétrole parle-t-on au Darfour? Si depuis 1999 l'exploitation pétrolière, massivement entre les mains d'intérêts chinois et malaisiens ${ }^{4}$, a bouleversé l'économie et la géopolitique soudanaise, elle concerne des concessions situées dans la partie méridionale du Soudan. Les présomptions de gisements au Darfour ne sont pas confirmées ${ }^{5}$.

13 Si la rente pétrolière soudanaise et la forte présence de l'Asie (devenue en quelques années le premier partenaire commercial du pays : à hauteur de $80 \%$ des exportations et 
de $34 \%$ des importations) mettent bel et bien le Soudan et donc la résolution du conflit du Darfour au centre de nouveaux enjeux internationaux, elles n'en sont pas responsables.

14 A l'absence d'information des premiers temps a donc succédé une multiplicité d'analyses, toutes pas également éclairées, certaines non dénués d'arrière-pensées idéologiques, qui loin d'aider à la compréhension de la crise en fixent trop souvent les limites au seul champ émotionnel. La dimension humanitaire de la crise a d'ailleurs été infiniment plus largement relayée par les médias que l'analyse de ses causes profondes ou des engagements diplomatiques en vue de sa résolution (Lavergne, 2005, p. 129).

Une première conséquence de cet «emballement» du débat a monopolisé le traitement politique et médiatique de la question du Darfour durant l'automne 2007. En effet, ce qui semble avoir motivé la singulière odyssée de l' "Arche de Zoé », en parallèle d'une étrange et naïve lecture des droits international et humanitaire et en plus d'une condescendance culturelle assez déconcertante, c'est l'indignation face à l'inaction et l'inefficacité supposées de l'ONU et des ONG présentes au Darfour.

Rien n'est moins vrai. Ces cinq dernières années ont au contraire vu le développement d'un dispositif d'assistance humanitaire sans précédent, qui a très certainement permis que soient épargnées des dizaines de milliers de vies et continue, en dépit de difficultés croissantes, d'éviter famines et épidémies majeures.

Ce sont aujourd'hui plus de 10000 travailleurs humanitaires qui portent assistance à près de quatre millions de personnes; le Programme Alimentaire Mondial et ses partenaires assurent la distribution de 25000 à 35000 tonnes chaque mois.

Bien sûr, le déploiement de l'aide est loin d'être parfait, et le satisfecit doit être nuancé ; au Darfour comme en d'autres lieux, l'humanitaire est d'ailleurs l'objet de très nombreuses critiques, sans doute autant dues à de réels dysfonctionnements qu'au fait que, à défaut de vraie solution, c'est lui qui « occupe l'espace ».

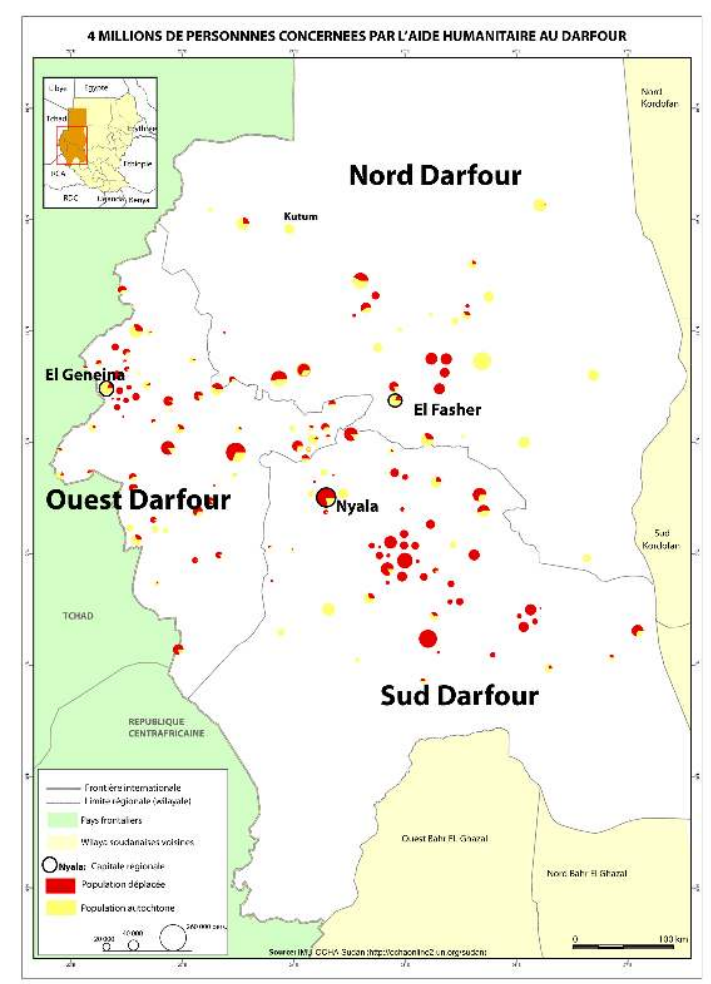

Au Darfour, précisément, tout l'espace n'est pas également occupé. Présentes par dizaines dans les principales villes de la région, 
qui offrent les conditions de travail les plus "confortables ", les ONG et plus encore les agences onusiennes sont significativement moins nombreuses dans les zones plus reculées, pourtant habitées : si nombre de déplacés ont trouvé refuge dans les camps installés en périphérie des principaux centres urbains, invariables illustrations de la crise humanitaire au Darfour, nombre d'autres ont formé d'autres types de rassemblement dans des villages préexistants, loin de toute ville d'importance. Certes moins assistés que les personnes ayant trouvé refuge dans les camps périurbains, ces déplacés-ci bénéficient néanmoins de l'aide, notamment alimentaire, et ne caractérisent pas une faillite du dispositif à répondre à l'essentiel des besoins.

Bindizi : distribution dans le village de Bindizi (Ouest Darfour) qui accueille 12000 déplacés

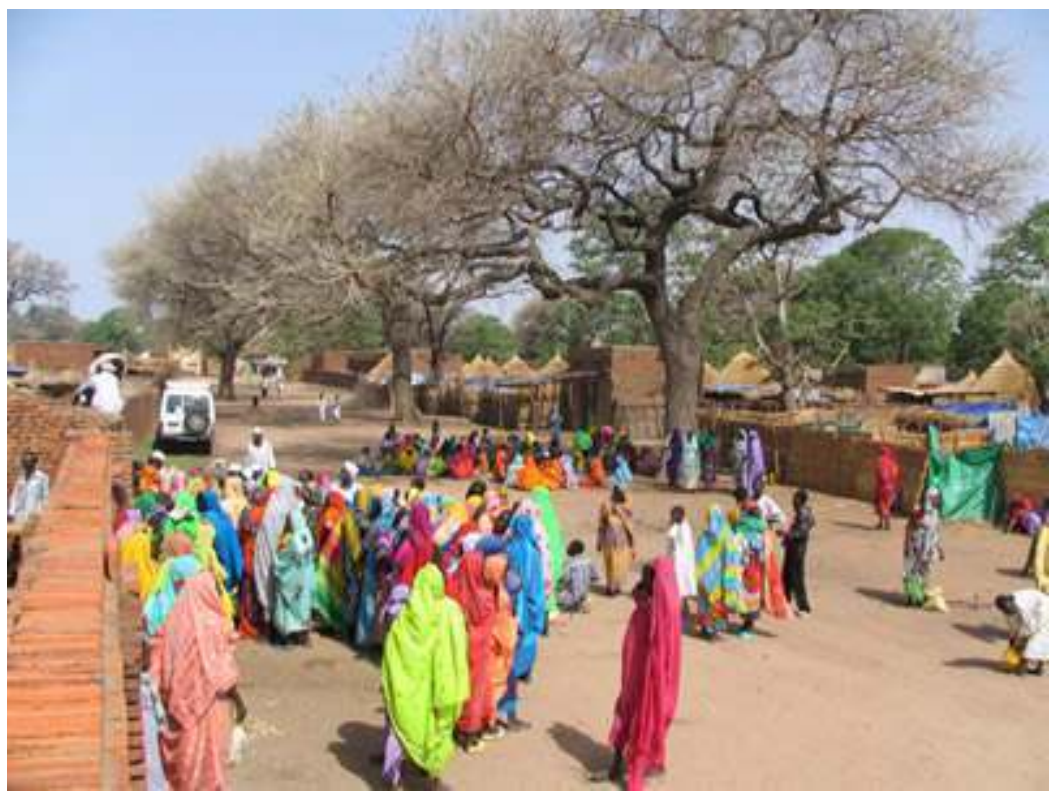

Cliché avril 2005

Latrines : alignement de latrines. Camp de Ryad (El Geneina, Ouest Darfour)

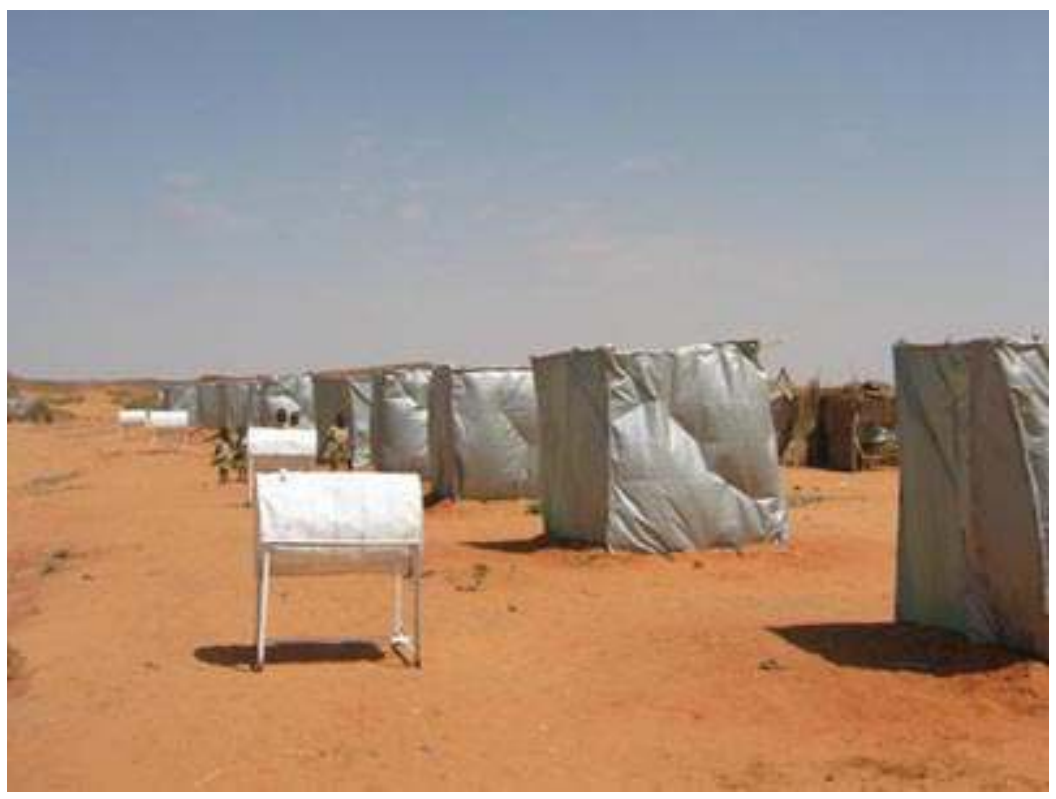




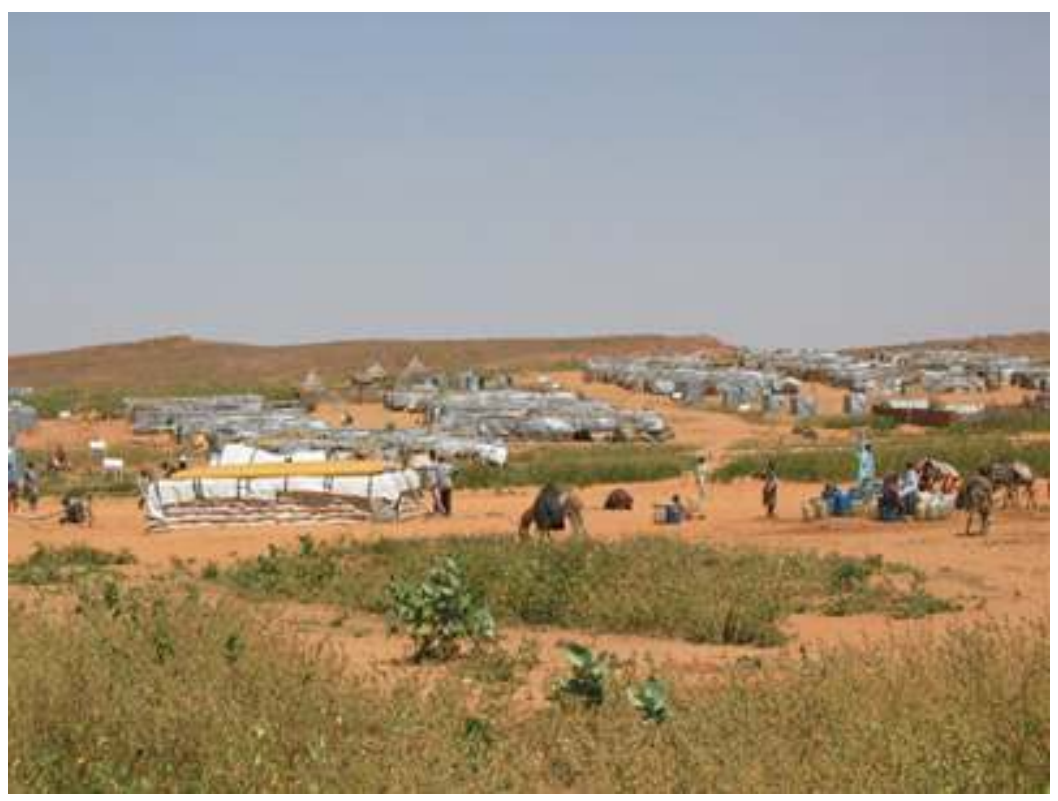

Un autre déséquilibre constaté de l'aide est qu'elle s'adresse encore peu aux populations non déplacées, pourtant elles aussi affectées par le conflit ${ }^{6}$. Les populations arabes notamment - mais pas exclusivement - sont encore peu considérées, comme si elles étaient tenues collectivement coupables des exactions commises par certains des leurs. Il est bien sûr normal que l'effort se soit d'abord concentré sur les populations déplacées, de loin les plus démunies; mais cinq années plus tard, celles-ci bénéficient d'un niveau de services qu'elles n'ont que rarement connu.

D'abord compréhensible, cette discrimination se comprend moins aujourd'hui, et constitue un risque de radicalisation d'individus (voire de communautés) restés jusque là à l'écart du conflit. Elle est déjà certainement un facteur de nouveaux déplacements, par l'attraction qu'exercent les camps et les services qu'ils proposent au regard des conditions de vie villageoises, rendues plus rudes encore par le dépeuplement et l'insécurité.

De fait, le dispositif d'aide humanitaire au Darfour procède malgré lui de la recomposition démographique et des processus d'urbanisation qui s'opèrent; en organisant et en améliorant les conditions d'accueil des populations rurales dans les camps périurbains, il risque de contribuer à leur pérennisation.

Similairement, l'ampleur de la présence humanitaire a directement contribué, conséquence certes non recherchée, à transfigurer les principales villes du Darfour, en en dynamisant les marchés de l'emploi, de biens (matériaux de construction, équipements... ), de services (transports, Internet...) ou immobilier (locations, constructions et réhabilitations d'immeubles...) : on construit au Darfour, et qui a vu el Geneina ou Nyala en 2004 serait frappé de la physionomie qu'ont ces villes aujourd'hui.

23 Non dénué d'effets collatéraux, critiquable à bien des égards, le dispositif humanitaire déployé au Darfour n'en a pas moins joué le rôle qui lui revenait; mais le traitement humanitaire de la crise est évidemment insuffisant et intervient d'abord au Darfour par défaut : il ne s'y maintient aujourd'hui qu'en l'attente d'une solution à la crise. 

au ceur de l'approche politique du conflit. Ainsi a-t-on vu, au printemps 2007 , mise en perspective l'organisation de "corridors humanitaires ", visant à permettre l'acheminement de l'aide au Darfour depuis la Centrafrique et le Tchad voisins. Or on l'a vu, l'aide arrivait déjà massivement au Darfour, et peut-être faut-il ne voir dans cette initiative française que l'introduction d'un débat ayant débouché sur la décision du déploiement de l'EUFOR ${ }^{7}$, très compatible avec les options politiques françaises au Tchad et en RCA. Unies (MINUAD), longuement et âprement négocié avec le gouvernement de Khartoum, accumule difficultés et retards, confirmant s'il était besoin qu'il ne saurait constituer une solution. Elle prend le relais de la mission de l'Union africaine au Darfour (AMIS) présente au Darfour depuis fin 2004 avec 7000 hommes. On peut raisonnablement douter que les 26000 soldats dont la présence est prévue à terme parviendront à sécuriser un aussi vaste territoire que le Darfour, quand en 199950000 soldats de l'OTAN ont été jugés nécessaires pour pacifier le Kosovo, territoire équivalent en surface à la Gironde.

Ces initiatives, dont le caractère " militaro humanitaire » affiché en rappelle d'autres, ont bien entendu le mérite d'exister, et valent évidemment mieux que la relative indifférence qui a accompagné les premiers mois du conflit. Mais elles semblent être devenues des objectifs en soi, et mobilisent de fait une large part des efforts diplomatiques, qui visent à lever les obstacles à leur mise en œuvre.

Dans le même temps, la perspective d'une solution politique s'éloigne. Le morcellement de la rébellion, spectaculairement révélé à Abuja, en avril 2006, par la signature d'un accord de paix par seulement l'un des chefs de la rébellion, a encore compliqué l'organisation de pourparlers. Les efforts en ce sens de la mission civile des Nations Unies impliquent une première reconnaissance formelle de groupes ou leaderships récents, officiellement conviés aux négociations, et radicalisent encore le refus d'y participer de chefs rebelles « historiques ", peu enclins à accepter cette nouvelle concurrence.

Parallèlement, le gouvernement Soudanais semble de moins en moins disposé à contribuer à une solution pacifique au conflit. En plus d'une capacité reconnue au double langage et aux revirements, le retentissement médiatique dont a fait l'objet la crise du Darfour et en particulier les accusations qui lui sont faites d'avoir planifié puis exécuté un " génocide », ont contribué à la radicalisation d'un gouvernement dont des membres sont par ailleurs menacés de poursuites pour les crimes commis.

Triste anniversaire donc.

31 Il est à craindre qu'il ne s'agisse pas du dernier. 


\section{BIBLIOGRAPHIE}

Denis E., 2005 (c), « Démographie ethnique, modernisation et violence au Darfour », in Annales d'Ethiopie, Vol. XXI, pp. 97-137

Lavergne M., 2005, «L'analyse géographique d'une guerre civile en milieu sahélien », in Afrique Contemporaine, Vol. 214, $\mathrm{n}^{\circ}$ 2, pp. 129-163

Prunier G., 2005, Le Darfour. Un génocide ambigu, Paris, La Table Ronde, 268 p.

Tubiana J., 2006, « Le Darfour, un conflit pour la terre? », in Politique Africaine, n 101 (avril), pp. 111-131.

vernochet J-M., 2007, « Le Darfour, de la crise politique au choc pétrolier », in Maghreb-Machrek, $\mathrm{n}$ - 193 (automne), pp. 55-77.

Flint J. et de Waal A., 2005, A Short History of a Long War, African arguments, 134p.

\section{NOTES}

1.Les accords de paix avec le Sud seront finalement signés en janvier 2005. Ce conflit aura été l'un des plus longs et des plus meurtriers de l'histoire de l'Afrique ; depuis la proclamation de l'Indépendance en janvier 1956, il n'aura connu qu'une interruption de 1972 à 1983.

2. Chiffres issus du pré-comptage organisé en novembre 2002 par le bureau de la statistique soudanaise en vue du recensement de 2003. Ils ne concernent que le Nord Soudan. La mise en œuvre du recensement a été retardée en prévision de l'accord de paix avec le sud de manière à couvrir l'ensemble du Soudan.

3. Ce contexte prévaut également pour la guerre dans le Sud.

4. « La chine a profité du boycott des démocraties occidentales à l'égard du Soudan pour investir massivement dans l'économie soudanaise et prendre le contrôle de l'exploitation pétrolière », (Denis, 2005, p. 106)

5. On évoque parfois aussi l'intérêt stratégique du Darfour dans la perspective d'un pipeline acheminant le pétrole tchadien jusqu'aux terminaux de Port Soudan, sur la Mer Rouge ; les 160000 barils/jour tchadiens sont déjà pompés jusqu'à la côte camerounaise, et on voit mal l'urgence qu'il y aurait à provoquer ou entretenir un conflit au Darfour pour permettre l'hypothétique construction d'un pipeline alternatif.

6. Les populations résidant dans les sites où sont venus se réfugier les personnes déplacées - les « communautés hôtes » - bénéficient néanmoins en général de l'aide alimentaire - cf. carte.

7. Pour "European Force", nom donné aux missions militaires de maintien de la paix issues de l'Union Européenne (Bosnie, Macédoine, etc.). Une mission de l'EUFOR est en cours de déploiement aux frontières est du Tchad et de la Centrafrique, dont la France fournit le principal contingent. 


\section{RÉSUMÉS}

La crise du Darfour entre dans sa sixième année. D'abord ignorée, elle fait aujourd'hui l'objet d'une importante couverture médiatique et de vives polémiques. Mais en dehors de l'assistance humanitaire portée aux personnes, la communauté internationale semble incapable d'imaginer une solution à une crise qui s'installe, et se complexifie.

The Darfur crisis entered its sixth year. At First almost ignored, it benefits today from a large coverage by Medias, and even became of source of disputes. However, apart of the relief aid provided to persons, the international community remains unable to find a solution to a lasting and more and more complicated crisis.

\section{INDEX}

Mots-clés : crise du Darfour, Soudan, humanitaire, diplomatie

Keywords : Darfur crisis, Sudan, humanitarian, diplomacy, EUFOR

\section{AUTEURS}

\section{ALICE FRANCK}

Alice Franck (alicefranck@yahoo.fr) est docteur en Géographie, Université Paris X Nanterre, Laboratoire Gecko.

\section{IVAN DERET}

Ivan Deret (ivan.deret@trianglegh.org) est chargé de programmes (association Triangle Génération Humanitaire). 